Case report

\title{
Pancreatic pseudocyst with pancreatolithiasis and intracystic hemorrhage treated with distal pancreatectomy: a case report Masato Maeda ${ }^{1}{ }^{*}$, Ryota Nomura ${ }^{1}$, Toshiaki Moriki ${ }^{2}$ and Tadashi Miyashita ${ }^{1}$
}

\author{
Addresses: ${ }^{1}$ Department of Surgery, Shizuoka City Shizuoka Hospital, 10-93 Ohtemachi Aoi-ku, Shizuoka 420-8630, Japan \\ ${ }^{2}$ Department of Pathology, Shizuoka City Shizuoka Hospital, 10-93 Ohtemachi Aoi-ku, Shizuoka 420-8630, Japan \\ Email: MM* - qqhxh715@ybb.ne.jp; RN - pulitzer@yahoo.co.jp; TMo - schopath@chabashira.co.jp; TMi - NCD01351@nifty.ne.jp \\ * Corresponding author
}

Received: 19 July 2009 Accepted: 29 July 2009 Published: 24 August 2009

Cases Journal 2009, 2:8693 doi: 10.4076/1757-1626-2-8693

This article is available from: http://casesjournal.com/casesjournal/article/view/8693

(C) 2009 Maeda et al.; licensee Cases Network Ltd.

This is an Open Access article distributed under the terms of the Creative Commons Attribution License (http://creativecommons.org/licenses/by/3.0), which permits unrestricted use, distribution, and reproduction in any medium, provided the original work is properly cited.

\begin{abstract}
Introduction: Hemorrhage from pancreatic pseudocyst is one of the serious complications of chronic pancreatitis. We experienced intracystic hemorrhage from a huge pancreatic pseudocyst and successfully treated it with distal pancreatectomy.

Case presentation: A 65-year-old-man with a history of alcohol abuse was admitted to our hospital for abdominal pain and was diagnosed as having chronic pancreatitis with pancreatolithiasis and pseudocyst in the pancreatic tail. The pancreatic pseudocyst increased in size gradually for 4 month observation period. For intracystic hemorrhage we performed an urgent distal pancreatectomy with splenectomy. Postoperative course was good and the elevated serum amylase level decreased to the normal range.

Conclusion: Prolonged observation resulted in intracystic hemorrhage. Drainage or surgery in adequate time is important for the management of pancreatic pseudocysts to prevent complications.
\end{abstract}

\section{Introduction}

Psuedocyst of the pancreas is a common and painful complication of chronic pancreatitis and complicates about $20 \%$ to $50 \%$ of patients after chronic pancreatitis [1]. Several complications of pancreatic pseudocysts have been reported, e.g., rupture, infection, biliary obstruction and hemorrhage. Hemorrhagic complications are expected in $6-31 \%$ of patients with pancreatic pseudocysts and can be life-threatening condition [2]. This article reports a case of intracystic hemorrhage from a huge pancreatic pseudocyst successfully treated with an urgent distal pancreatectomy.

\section{Case presentation}

A 65-year-old Japanese man was admitted to our hospital in April 2007 for abdominal pain after drinking alcohol. The patient details are as follows: Occupation: company employee; Ethnicity: Japanese; Weight: 45 kg; Height: $157 \mathrm{~cm}$; Medical history: no appreciable disease ; Family history: unremarkable ; Habits: $900 \mathrm{ml}$ of Japanese Sake (corresponding to $1,200 \mathrm{ml}$ of wine) per day for 45 years and 20 cigarettes per day for 45 years. Abdominal examination carried out on the 1st consultation showed mild tenderness on the epigastric region without significant rebound and a palpable mass. The reminder of his 
examination was unremarkable. Laboratory tests showed the following values: amylase, $811 \mathrm{IU} / \mathrm{L}$ (reference level, 40-120); lipase, 732 IU/L (reference level, 17-57); Elastase$1,2811 \mathrm{ng} / \mathrm{ml}$ (reference level 72-432); C-reactive protein, 3.24 (reference level 0.00-0.30). Values of other blood chemistry and hematology were unremarkable. An abdominal computed tomography (CT) scan showed many stones in the main pancreatic duct and a cystic lesion, $4 \mathrm{~cm}$ diameter, located in the pancreatic tail, that was diagnosed as pancreatic pseudocyst with pancreatolithiasis accompanied with chronic pancreatitis (Figure 1).

Initially the patient was treated conservatively but 6 weeks later abdominal pain had become severe and the cyst
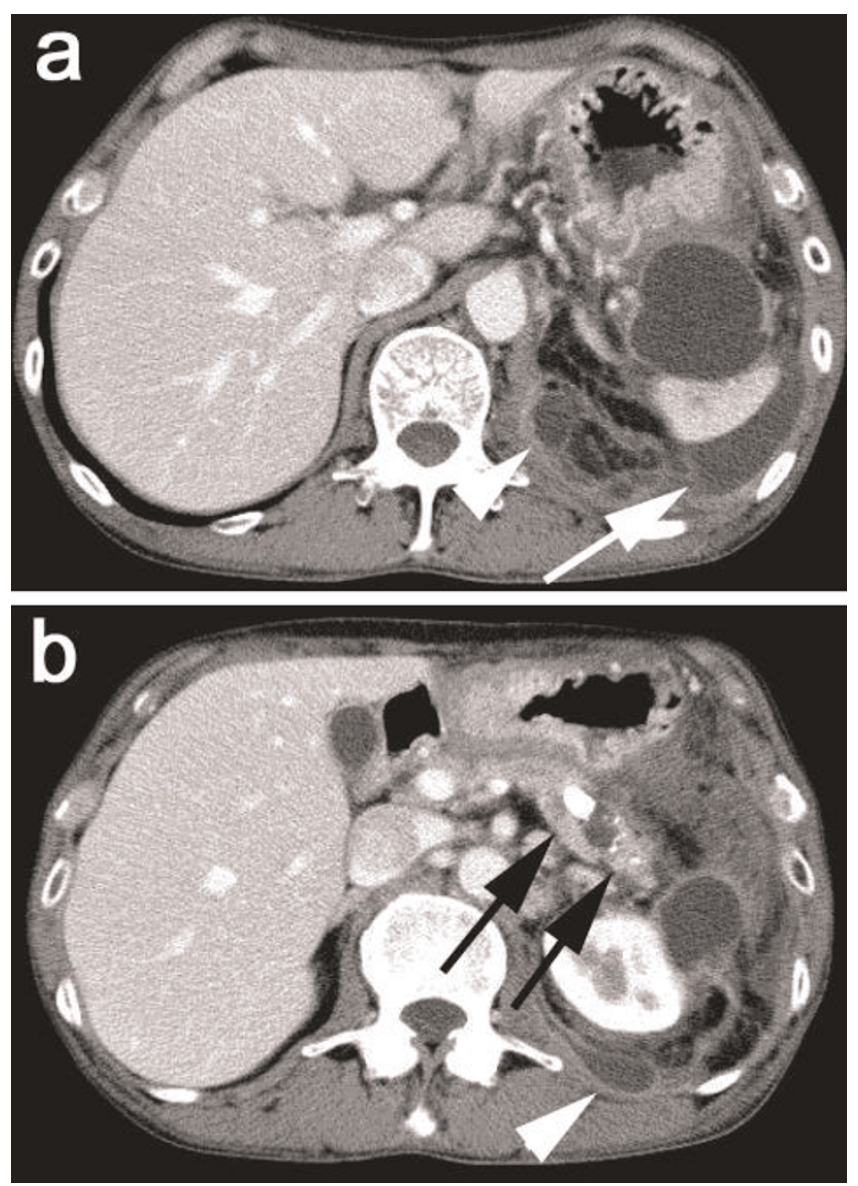

Figure I. An abdominal CT scan at the Ist consultation showed many stones in the main pancreatic duct (black arrows in b) and a cystic lesion, $4 \mathrm{~cm}$ diameter, located in the pancreatic tail and surrounded by the stomach, the spleen and the left kidney. The cystic lesion extended to the dorsal side of the left kidney (white arrowhead in b) and there was small amount of fluid collection around the spleen, which was considered to be caused by acute inflammation on chronic pancreatitis (white arrow in a). increased in size upto $6 \mathrm{~cm}$ diameter on CT scan. We recommended surgery, but the patient refused to undergo an operation and hoped medical management. Treatment using gabexate mesilate diminished his symptom but did not decrease the serum amylase level. 4 months after his first consultation abdominal pain became severe again and the cyst increased in size (Figure 2).

Once his symptom was relieved, after several days he complained a sudden and more severe left upper quadrant pain. Ultrasonography examination revealed heterogeneous mass in the cyst, which suggested intracystic hemorrhage (Figure 3).

CT scan also demonstrated high density structure in the cyst, which had not been found out on the previous CT scan, but showed no aneurysm or extravasation apparently (Figure 4).

Although the patient showed no sighs of shock, we diagnosed it as an intracystic hemorrhage and immediately selective angiography examination (SAG) was performed. SAG from celiac trunk or splenic artery demonstrated no aneurysm or extravasation (Figure 5a) but showed complete obstruction of the splenic vein and a collateral formation in the greater curvature side of the stomach (Figure 5b).

Then we performed an urgent operation, which disclosed that the cyst of diameter $7 \mathrm{~cm}$ size involving the pancreatic tail and the spleen adhered firmly to the stomach and the colon, and the lower part of the cyst wall was united with a circumference fascia of the left kidney. A distal pancreatectomy with splenectomy was performed to remove all the stones in the pancreas and the cyst wall was removed partially to preserve the stomach, the colon and the left kidney (Figure 6).

The content of the cyst was dark reddish-brown bloody liquid and blood coagula. The value of amylase and CA19-9 of the liquid was $48,520 \mathrm{U} / \mathrm{L}$ and $7,880 \mathrm{U} / \mathrm{ml}$, respectively. Histopathological finding revealed that no epithelium was observed in the inner lining and fibrosis and infiltration of inflammatory cells in the surrounding pancreatic tissue with atrophy was observed, then it was diagnosed as a chronic pancreatitis with a pseudocyst without any malignant portion. Postoperative course was uneventful and the serum amylase value decreased to the normal range, then the patient was discharged on postoperative day 18 .

\section{Discussion}

A pancreatic pseudocyst is a localized fluid collection within or adjacent to the pancreas, enclosed by a nonepithelialized wall. As acute pseudocysts are known to 

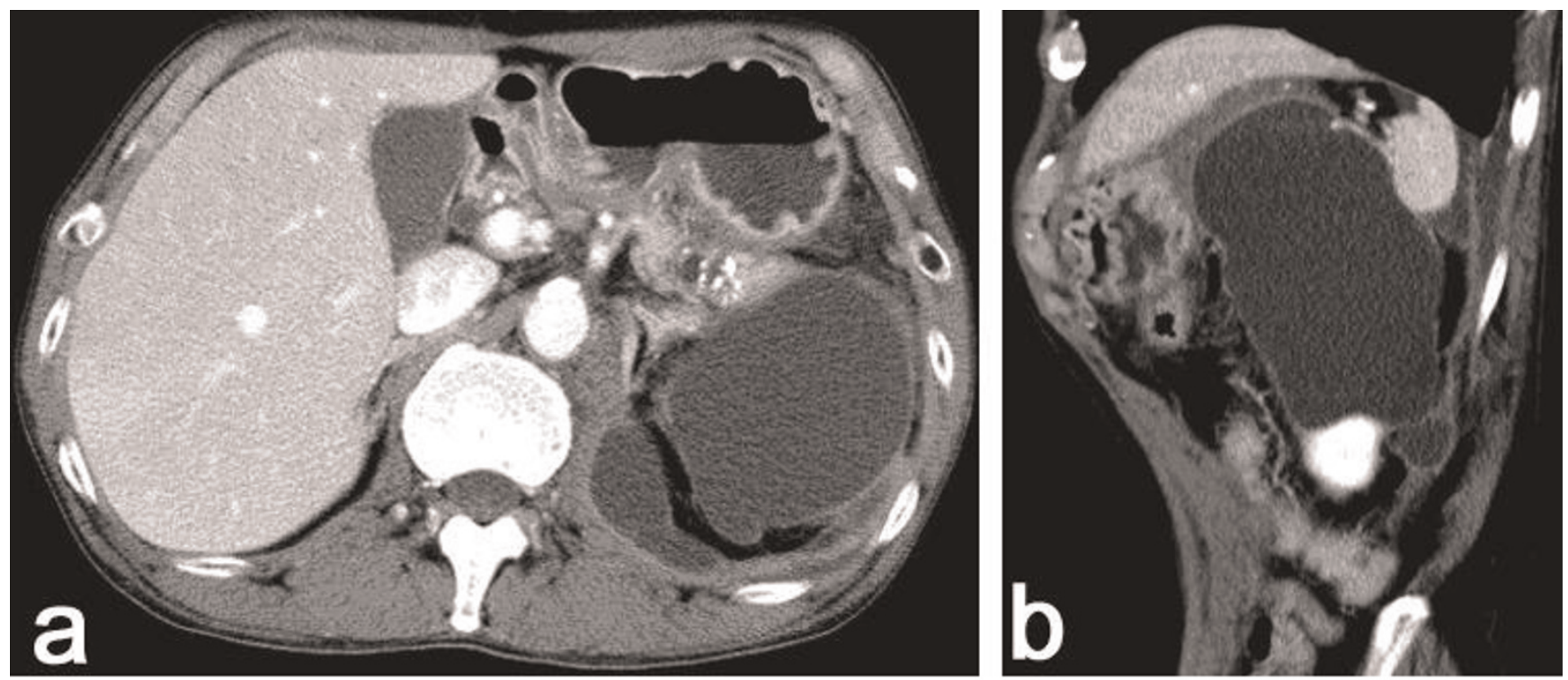

Figure 2. After 4 months conservative treatment CT scan demonstrated the cyst increased in size to $7 \mathrm{~cm}$ diameter on horizontal section (a) and $\mathrm{II} \mathrm{cm}$ in the cranio-caudal direction (b).

resolve spontaneously in considerable frequency, expectant management for at least 4 to 6 weeks should precede surgery or intervention [3]. In contrast chronic pseudocysts rarely regress if they are larger than 4 to $6 \mathrm{~cm}$ in diameter [2]. As the pancreatic pseudocyst in our case, which was accompanied with an alcoholic chronic pancreatitis, was referred to a chronic pseudocyst, spontaneous regression was hardly expected. In addition the stones in the main pancreatic duct were considered to disturb the flow of the pancreatic juice toward the duodenum and allow the cyst
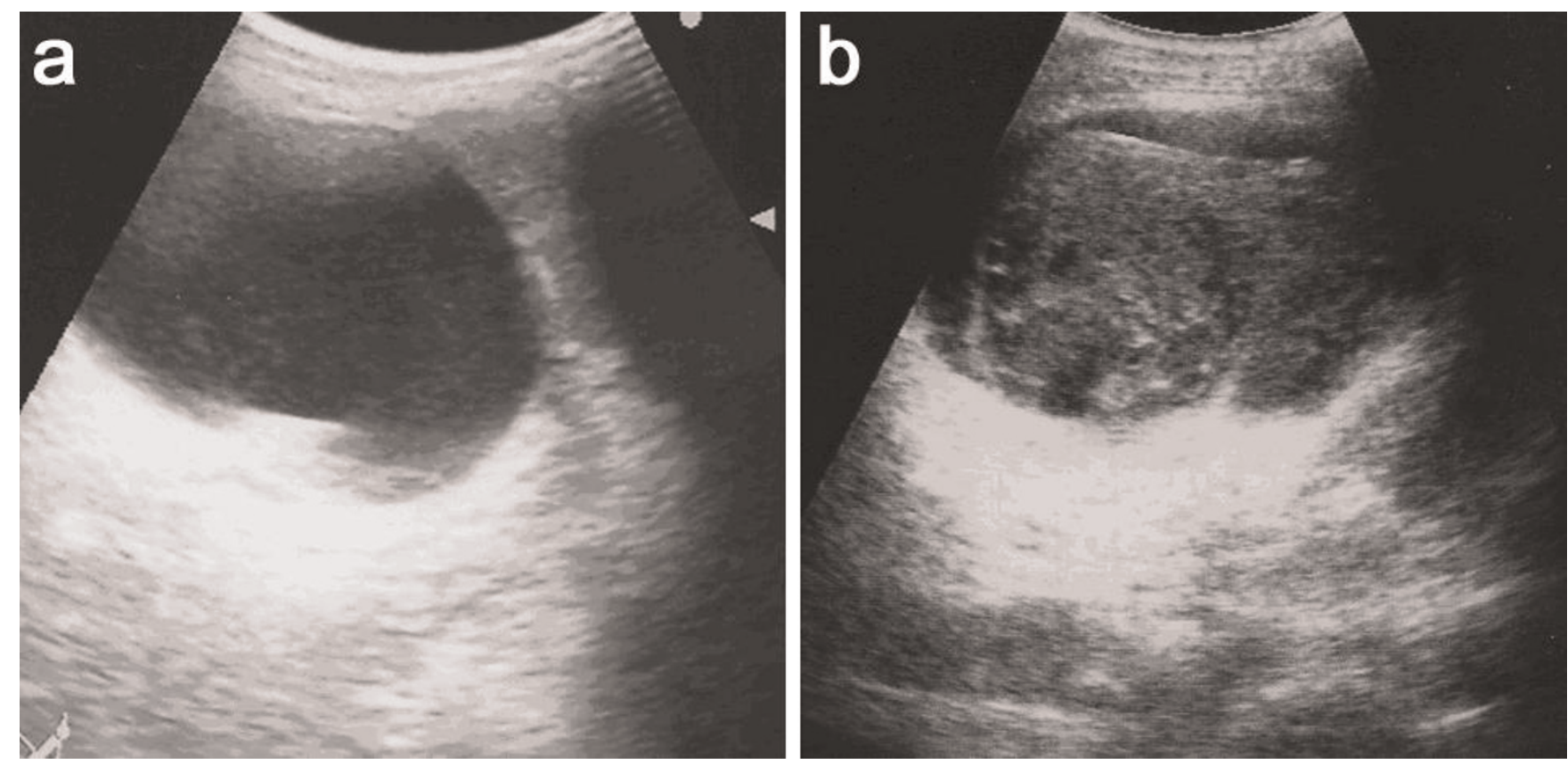

Figure 3. Ultrasonography examination revealed heterogeneous mass in the cyst (b), which had not been found 3 days before (a). 


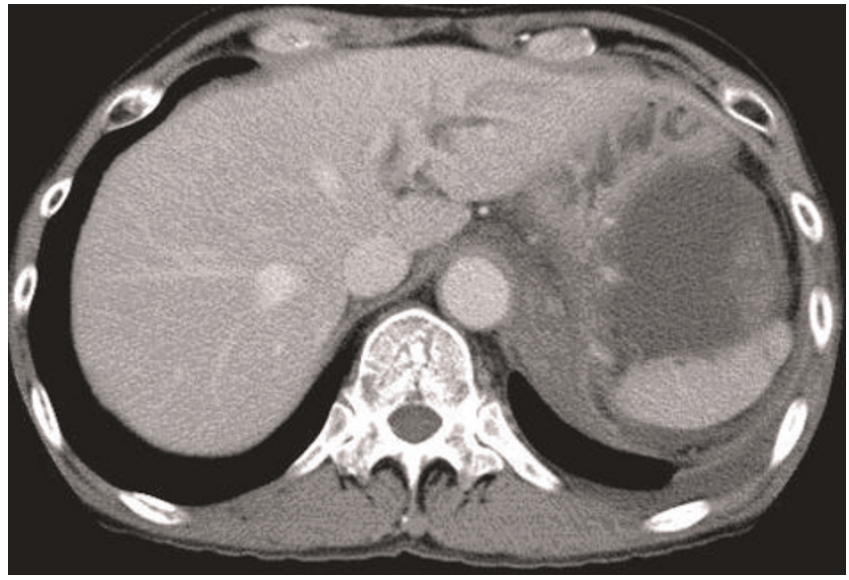

Figure 4. CT scan demonstrated high density structure in the cyst.

to become gradually greater. Acute hemorrhage from a pseudocyst is one of the most serious complications of chronic pancreatitis. The sudden onset of an abdominal pain was considered by rapidly increased intracystic pressure due to acute hemorrhage. The progressive enlargement of the pseudocyst induces necrotizing vessels because of the added pressure on the vessel walls and from the action of proteolytic enzymes. This leads to the erosion of the vessel walls, resulting in intracystic hemorrhage [4]. Another important cause of intracystic hemorrhage is the rupture of the pseudoaneurysm, which often results in massive bleeding to cause shock status and is usually treated by emergency transarterial embolization (TAE) or operation [5]. As no pseudoaneurysm or extravasation was detected on the angiography examination, we performed urgent operation without TAE.

The accepted opinion about the timing of treatment for pseudocysts has been as follows: (i) a pseudocyst that occurs after an episode of alcohol-related pancreatitis has to be observed for 4 to 6 weeks. (ii) After 6 weeks observation should continue if the size of the cyst is less than $6 \mathrm{~cm}$ and the patient is asymptomatic or if there is decrease in size. (iii) Therapy is indicated if the patient is symptomatic or if the cyst size is more than $6 \mathrm{~cm}$, the cyst is increasing in size, the cyst is infected, or there is a suspicion of malignancy. (iv) Observation is unnecessary, and immediate drainage is safe in cysts that have a mature wall or in those arising in chronic pancreatitis [6]. Percutaneous drainage of cysts is the least invasive method, however it is not the procedure of the choice in the presence of a drainage disturbance in the main pancreatic duct like our case, because of the risk of a permanent external fistula [6]. Percutaneous and trans gastric puncture of pancreatic cysts to make cystogastrostomy guided by ultrasonogtaphy and gastroscopy, has the advantage of establishing an internal fistula between the cyst and the stomach and preventing a pancreatic cutaneous fistula [7]. Endoscopic drainage is also less invasive and makes internal fistula to achieve a low recurrence rate As operative methods cystgastrostomy or cystjejunostomy to make internal drainage is often performed and recently it may be done laparoscopically [8]. However we have to pay attention to the fact that massive hemorrhage can occur not only in untreated pseudocysts but also following internal drainage [9]. Although resection of cysts is usually accompanied with
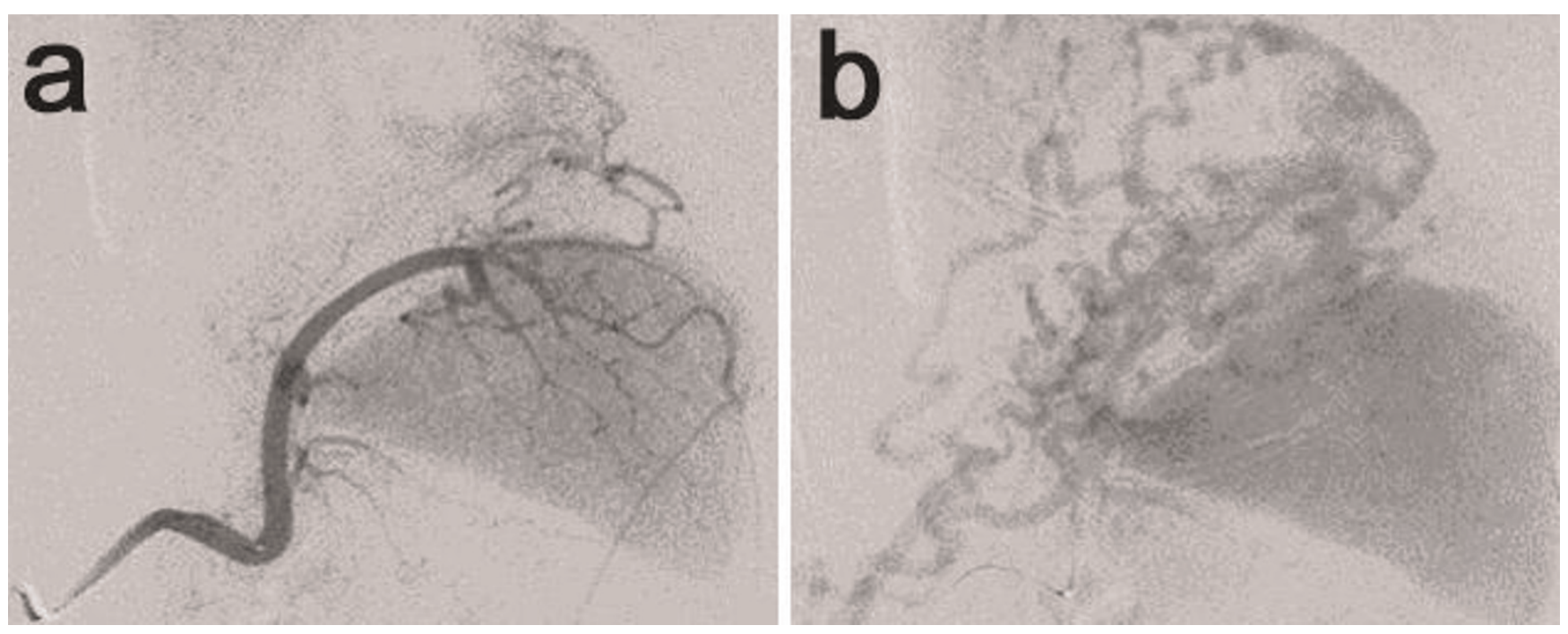

Figure 5. Angiography examination from the splenic artery demonstrated no aneurysm or extravasation (a) and showed a collateral formation in the greater curvature side of the stomach instead of the splenic vein in the late phase (b). 

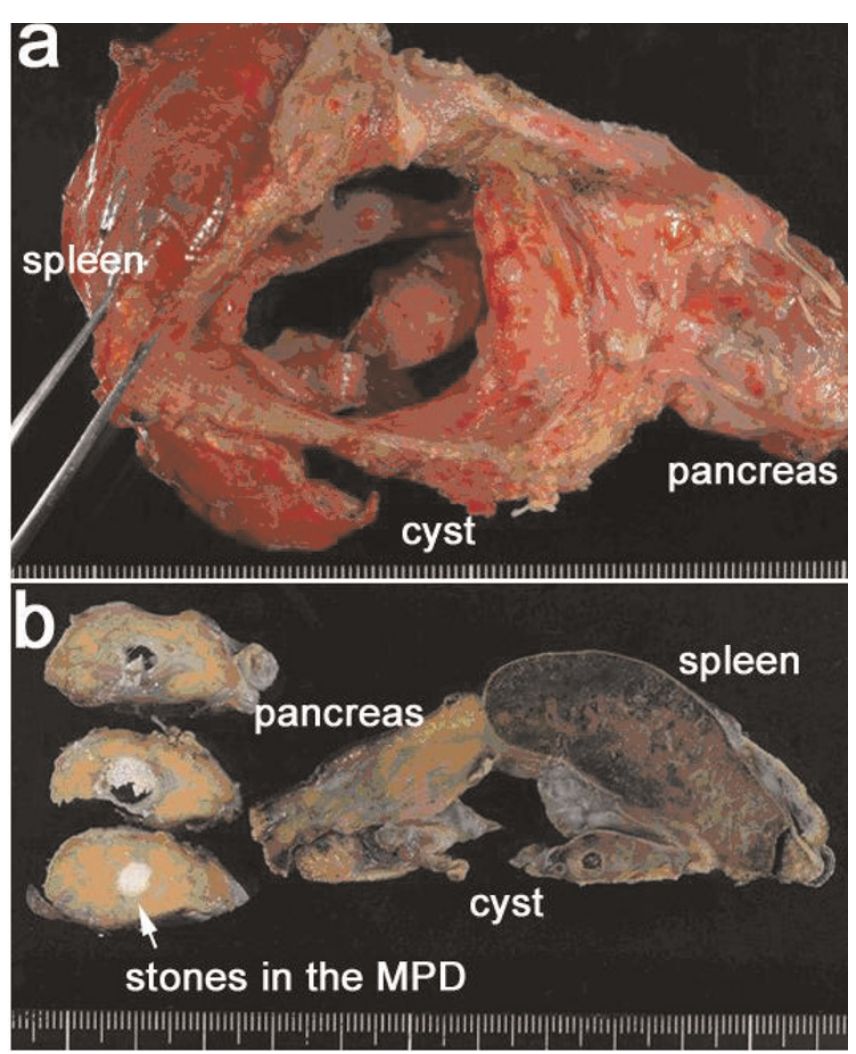

Figure 6. Raw resected specimen was observed from the dorsal side (a). Sagittal section (left side of $\mathbf{b}$ ) and coronal section (right side of $\mathbf{b}$ ) of formalin fixed specimen. MPD; main pancreatic duct.

pancreatic resection and is more invasive method, the episode of intracystic hemorrhage made the choice of anastomosis of the cyst to the alimentary tract risky for recurrent hemorrhage and a removal of the pancreatic stones as well as the cyst by pancreatic resection provided definitive control [10].

\section{Conclusion}

Chronic pancreatic pseudocyst rarely regress if they are larger than 4 to $6 \mathrm{~cm}$ in diameter. As the patient had initially refused an operation, conservative treatment was performed for a too long period in our case, that allowed the cyst growing and hemorrhagic complication. Drainage or surgery in adequate time is important for the management of pancreatic pseudocysts to prevent complications.

\section{Abbreviations}

CA19-9, carbohydrate antigen 19-9; CT, computed tomography; SAG, selective angiography; TAE, transarterial embolization.

\section{Consent}

Written informed consent was obtained from yhe patient for publication of this case report and accompanying images. A copy of the written consent is available for review by the Editor-in-Chief of this journal.

\section{Competing interests}

The authors declare that they have no competing interests.

\section{Authors' contributions}

MM participated in the patient's treatment, conceived the case report and contributed in the first draft, $\mathrm{RN}$ participated in the patient's treatment and collected patient's data, $\mathrm{TM}^{2}$ contributed pathological diagnosis, $\mathrm{TM}^{1}$ supervised the patient's treatment and the manuscript. All authors have read and approved the final manuscript.

\section{References}

I. Ho HS, Frey CF: Current approach to the surgical management of chronic pancreatitis. Gastroenterologist 1997, 5: 128-136.

2. Sankaran S, Walt AJ: The natural and unnatural history of pancreatic pseudocysts. Br J Surg 1975, 62:37-44.

3. Crass RA, Way LW: Acute and chronic pancreatic pseudocysts are different. Am J Surgery 198I, I42:660-663.

4. Mansueto G, Cenzi D, D’Onofrio M, Salvia R, Gottin L, Gumbs AA, Mucelli RP: Endovascular treatment of arterial bleeding in patients with pancreatitis. Pancreatology 2007, 7:360-369.

5. Gombiez LP, Ernst OJ, Merlier OA, Porte HL, Chambon JP, Quandalle PA: Arterial embolization for bleeding pseudocysts complicating chronic pancreatitis. Arch Surg 1997, I32:10161021 .

6. Pitchumoni CS, Agarwal N: Pancreatic pseudocysts. When and how should drainage be performed? Gastroenterol Clin North Am 1999, 28:615-639.

7. Hancke S, Henriksen FW: Percutaneous pancreatic cystogastrostomy guided by ultrasound scanning and gastroscopy. $\mathrm{Br}$ J Surg $1985, \mathbf{7 2 : 9 1 6 - 9 1 7 .}$

8. Aljarabah M, Ammori BJ: Laparoscopic and endoscopic approaches for drainage of pancreatic pseudocysts: a systematic review of published series. Surg Endosc 2007, 2 1:19361944.

9. Ikoma A, Tanaka K, Ishibe R, Ishizaki N, Taira A: Late massive hemorrhage following cystogastrostomy for pancreatic pseudocyst: report of a case. Surg Today 1995, 25:79-82.

10. Bender JS, Bouwman DL, Levison MA, Weaver DW: Pseudocysts and pseudoaneurysms: surgical strategy. Pancreas 1995, 10:143147. 